\title{
Segnalazione di una nuova popolazione di Dolichopoda schiavazzii caprai presente nella grotta Buca Tana di Maggiano (Lucca, Toscana, Italia) con alcune note biogeografiche sulla diffusione della specie in Toscana \\ (Orthoptera, Rhaphidophoridae)
}

\begin{abstract}
Riassunto: Viene segnalata una nuova popolazione di Dolichopoda schiavazzii individuata nella grotta Buca Tana di Maggiano nel comune di Lucca (Toscana), alle pendici meridionali delle Alpi Apuane. Sono inoltre riportate alcune osservazioni sulla diffusione della specie nella Toscana nord-occidentale alla luce delle attuali conoscenze dell'evoluzione paleoambientale e paleoclimatica della regione.

Abstract: New population of Dolichopoda schiavazzii caprai from the Buca Tana di Maggiano cave (Lucca, Tuscany, Italy) with some biogeographical notes about the spread of this species in Tuscany. (Orthoptera, Rhaphidophoridae)

A new population of Dolichopoda schiavazzii caprai Lanza, 1954 identified in the cave Buca Tana di Maggiano (Lucca, Tuscany) in southern foothills of the Apuan Alps is described. Moreover some observations on the spread of the species in Tuscany based on the paleoclimatic and paleoenvironmental evolution of the region where the discovery was made are given.
\end{abstract}

Key words: Rhaphidophoridae, Dolichopoda, Lucca (Tuscany), paleogeographic events, biogeography.

\section{INTRODUZIONE}

Il genere Dolichopoda (Orthoptera, Rhaphidophoridae) comprende una cinquantina di specie distribuite in un areale perimediterraneo che dalle montagne del Caucaso, attraverso l'Anatolia, la penisola greca e le isole dell'Egeo, raggiunge l'Italia, (dalle Alpi alla Calabria), la Sardegna, la Corsica e la parte orientale dei Pirenei. Tutte le Dolichopoda conducono parte del loro ciclo biologico all'interno di cavità naturali o artificiali e raramente si osservano esemplari in ambiente epigeo. In Italia sono presenti nove specie e cinque sottospecie, mentre in Toscana si trovano, da nord verso sud: $D$. laetitiae laetitiae $\mathrm{Mi}$ nozzi, 1920 nelle province di Lucca, Pistoia, Prato, Firenze, Siena, Arezzo; D. laetitiae etrusca Baccetti \& Capra, 1959 nella Maremma Grossetana e al Monte Amiata; D. baccettii Capra, 1957 al Monte Argentario (Prov. Grosseto) e D. aegilion Baccetti, 1975 esclusiva dell'Isola del Giglio (Prov. Grosseto). D. schiavazzii, descritta per la prima volta da Capra (1934) sugli esemplari provenienti dagli acquedotti del Limone e di Colognole a Livorno, fu dedicata al professor Giuseppe Schiavazzi, sismologo, entomologo e tassidermista di origini piemontesi ma livornese di adozione.
La sottospecie nominale D. schiavazzii schiavazzi Capra, 1934 è presente nelle province di Grosseto, Siena, Pisa, Livorno, mentre la sottospecie caprai Lanza, 1954 era conosciuta sino ad oggi esclusivamente nella caverna del Fichino presso Casciana Terme (provincia di Pisa).

\section{AREA DI STUDIO}

La Buca Tana di Maggiano è una cavità naturale posta, alla quota di 160 metri s.l.m., sulle colline prospicienti la frazione di Maggiano (Comune di Lucca, LU), a poca distanza dalla strada regionale 439 Sarzanese-Valdera che da Lucca si dirige verso il valico del Monte Quiesa (coordinate geografiche $43^{\circ} 51^{\prime} 31^{\prime \prime} \mathrm{N}-10^{\circ} 24^{\prime} 09^{\prime \prime} \mathrm{E}$, indicata come T/LU 827 nel catasto delle grotte della Toscana). L'ingresso della grotta, non molto ampio ma di agevole accesso, si apre alla base di un declivio roccioso in un lecceta naturale alle pendici SE del Monte dei Frati, sulla sponda sinistra del Rio della Selvarella. La cavità si sviluppa per una ottantina di metri all'interno della cosiddetta Formazione della Maiolica che, geologicamente, appartiene al Dominio Toscano e precisamente alla Falda Toscana. Il dislivello tra l'ingresso della grotta e il suo

\footnotetext{
${ }^{*}$ Alessandro Bramanti, Via Salesiani 25, 55045, Pietrasanta (LU), Italia. E-mail: alessandrobramanti@libero.it
} 
fondo cieco è di circa sedici metri, mentre in certi punti l'altezza raggiunge i 10 metri e oltre. La temperatura interna, rilevata nel mese di maggio 2016, oscillava tra i $18.4^{\circ} \mathrm{C}$ dell' ingresso e i $15.1^{\circ} \mathrm{C}$ della parte più interna con una media di $16.6^{\circ} \mathrm{C}$. La fauna entomologica della Buca Tana di Maggiano è stata indagata a partire dagli inizi del secolo scorso quando vi fu descritto per la prima volta il coleottero Duvalius jureceki rasettii Dodero, 1917. Altre ricerche biospeleologiche furono compiute da Lazzeroni (1970), Vanni (1987) e, recentemente, da Magrini \& Abbazzi (2011) senza però mai far menzione delle specie di ortotteri presenti.

Durante una recente visita a questa cavità, compiuta nel dicembre del 2015, la mia attenzione è stata attirata dalla presenza di numerosi esemplari di ortotteri appartenenti al genere Dolichopoda e alla specie Gryllomorpha dalmatina Ocskay, 1932 . Gli individui delle due specie, sia adulti che neanidi, sono stati osservati sino a una decina di metri di profondità sulle pareti della grotta caratterizzate da rientranze e anfratti che seguono la stratificazione e le discordanze della roccia. Conoscendo le peculiarità delle specie appartenenti al genere Dolichopoda, che si ritrovano tutte in ambienti ipogei naturali o artificiali, e la loro importanza dal punto di vista biogeografico, ho pensato di compiere, successivamente alla prima visita, un campionamento di esemplari, sia maschi sia femmine adulti, per poter osservare i loro caratteri morfologici al microscopio ottico e giungere così ad una attribuzione specifica.

Materiale esaminato: $2 \propto q, 08.12 .2015$, Toscana, Maggiano (LU), Bucatana di Maggiano, A. Bramanti leg.; 3 우 우 subadulte, Toscana, Maggiano (LU), Bu-

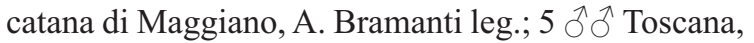
Maggiano (LU), Bucatana di Maggiano, A. Bramanti leg.; 2 우 13.12.2015, Toscana, Maggiano (LU), Bucatana di Maggiano, A. Bramanti leg.; 3 우 subadulte, 13.12.2015, Toscana, Maggiano (LU), Bucatana di Maggiano, A. Bramanti leg.: 3 $\widehat{\jmath} \widehat{\jmath}, 13.12 .2015$, Toscana, Maggiano (LU), Bucatana di Maggiano, leg. A. Bramanti.

\section{IDENTIFICAZIONE}

Ad una analisi preliminare gli esemplari raccolti alla Buca Tana di Maggiano presentano l'aspetto tipico del genere Dolichopoda: corpo slanciato dalle lunghe zampe, convesso dorsalmente, assenza di ali
(Fig. 1). Le dimensioni del corpo variano tra 13 e 20 $\mathrm{mm}$, le femmine di solito sono più grandi dei maschi (dimensioni 17-20 mm); le antenne sono molto lunghe, almeno tre volte la lunghezza del corpo (59-65 $\mathrm{mm}$ ). Il colore generale è giallo verdastro con la parte posteriore di tutti segmenti, dal mesonoto ai tergiti addominali, più scura con caratteristico aspetto $a$ fasce; le femmine hanno tonalità testacea (Fig. 2). Ventralmente tutti gli esemplari sono biancastri compresa la parte inferiore delle zampe. L'attribuzione alla specie D. schiavazzii è confermata dall'osservazione del $\mathrm{X}$ tergite del maschio e dell'epifallo (Fig. 3A,B,G) confrontati con i dati riportati in letteratura (Baccetti, 1976, fig. 3; Di Russo \& Rampini, 2012, tav. 67, fig. 3 e tav. 69, fig. 1). Le differenze risultano minime, comprese nell'ambito della variabilità della specie. Le femmine presentano un ovopositore lungo in media $12-13 \mathrm{~mm}$ dotato di circa 20 denticoli apicali nelle valve inferiori (Fig. 3D; vedi Baccetti, 1976, fig. 2c), la placca sottogenitale, dal margine posteriore arrotondato, in zona mediana, presenta un rigonfiamento pro-

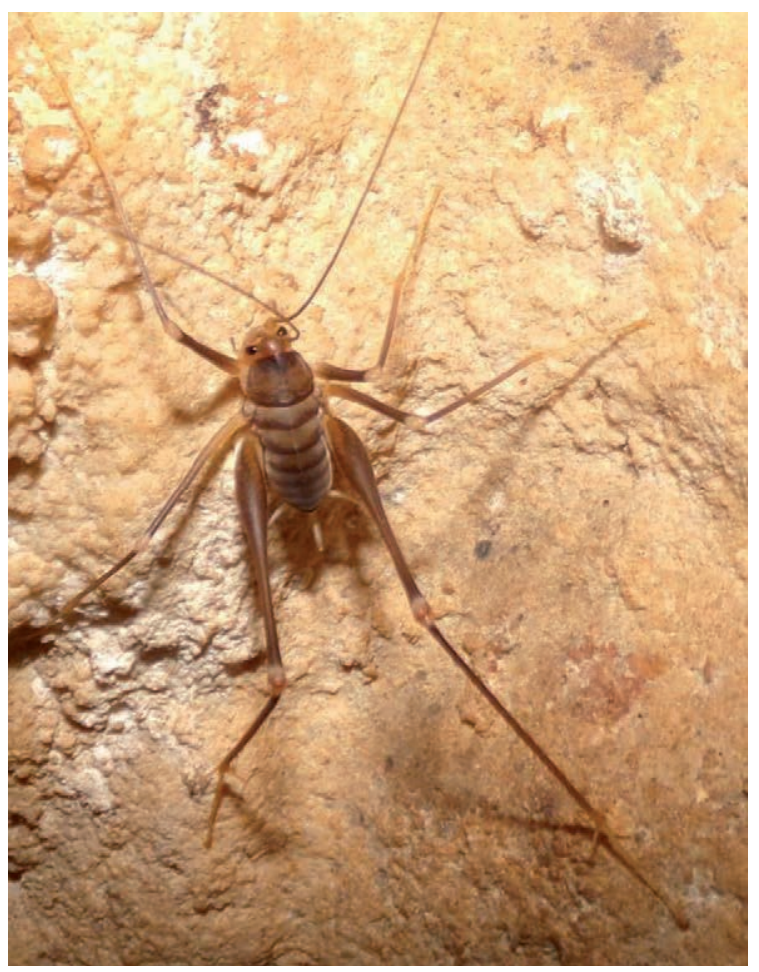

Fig. 1. Un esemplare di Dolichopoda schiavazzii caprai della Buca Tana di Maggiano (foto dell'autore). 
nunciato, troncato anteriormente (Fig. 3C). Infine l'osservazione della spinulazione delle zampe, in particolare dei femori mediani, ha permesso di atttribuire gli esemplari di Dolichopoda schiavazzii della Buca Tana di Maggiano alla ssp. caprai fino ad ora conosciuta unicamente per la Grotta del Fichino di Casciana Terme (Pi) (Lanza, 1954, 1957). Nella Dolichopoda schiavazzi schiavazzii i femori mediani possiedono 18-24 spine disposte su due file parallele mentre nella popolazione della Bucatana i maschi non hanno spine e solo le femmine possono avere da 1 a 3 spine in vicinanza delle geniculazioni (Fig. 3E,F). La distinzione tra D. schiavazzii schiavazzii "dai femori medi abbondantemente spinulosi lungo i margini inferiori" e $D$. schiavazzii caprai "con i femori medi inermi o muniti di rare spinule lungo il margine inferiore" (Baccetti,

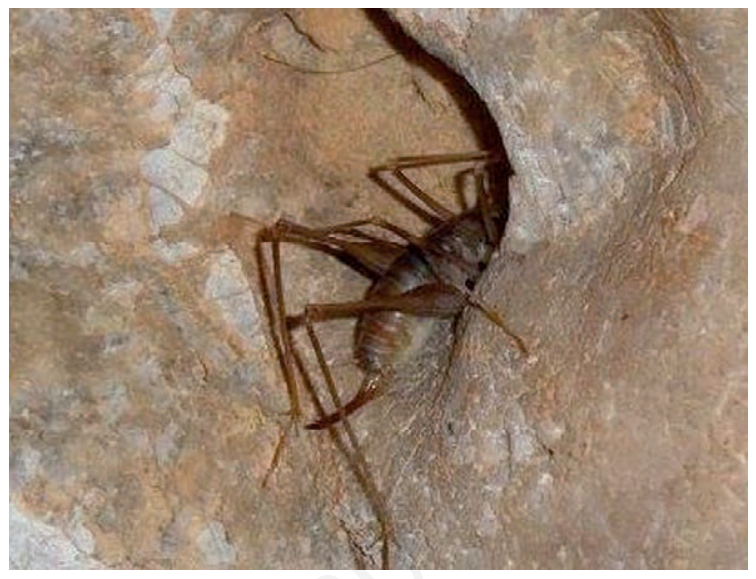

Fig. 2. Una femmina adulta di Dolichopoda schiavazzii caprai si nasconde in un anfratto della roccia (foto dell'autore).

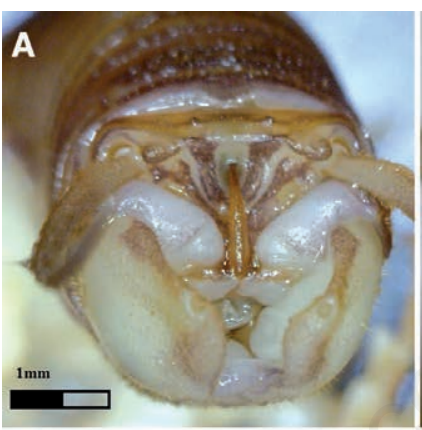

D
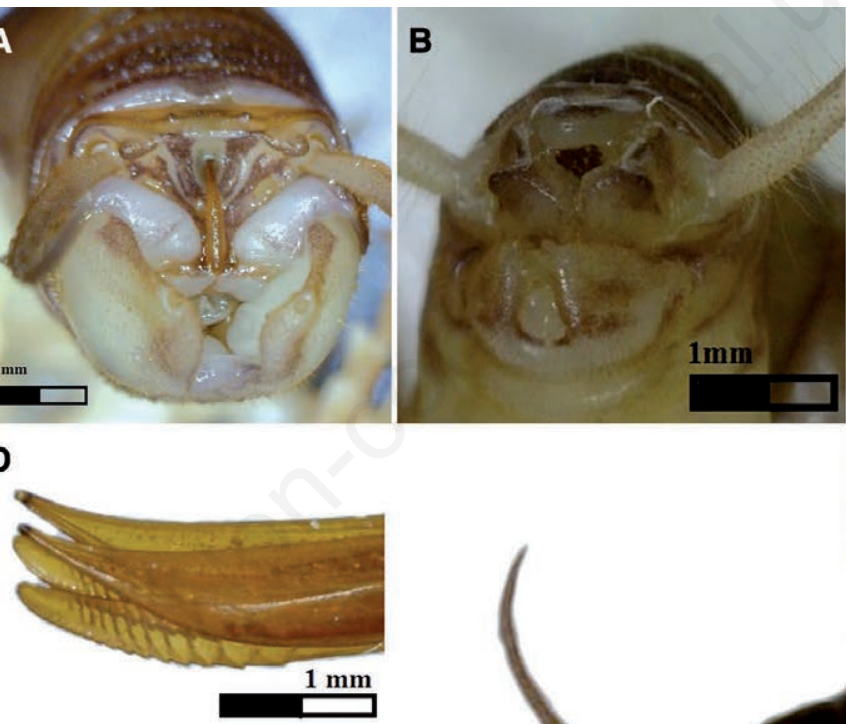

E

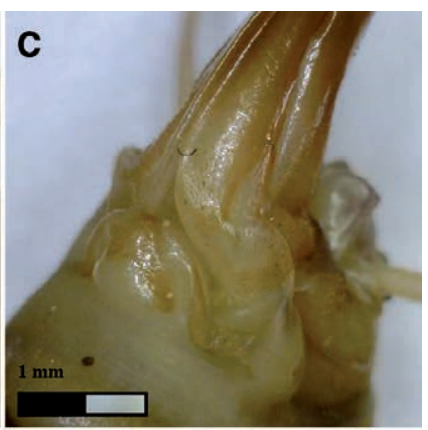

E
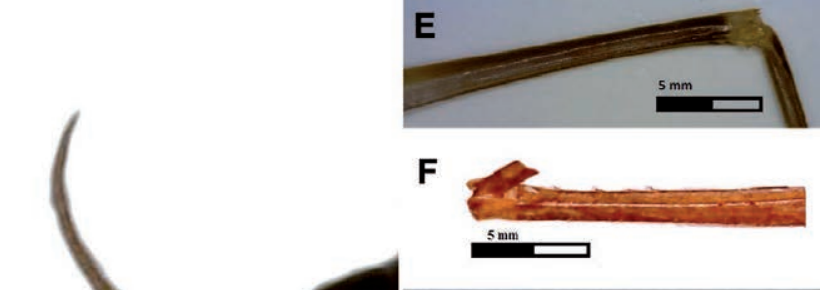

$\mathbf{F}$
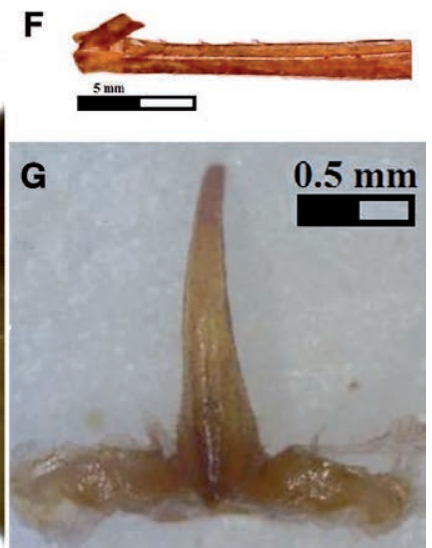

Fig. 3. Dolichopoda schiavazzii caprai; A) đ̊ addome, visione posteriore; B) $\widehat{\partial}$ addome, placca sottogenitale, paraprocti e epiprocto; C) $q$ placca sottogenitale; D) $q$ ovopositore e dentelli delle valve inferiori; E) $\widehat{\partial}$ femore mediano senza spinulazioni; F) $\rightarrow$ femore mediano con rade spinulazioni; G) ô epifallo, visione dorsale. 
1966, p. 24) è chiara e anche Di Russo \& Rampini (2012, p. 322) ribadiscono "la scarsa spinulazione dei femori medi, da 0 a 5 spine nei maschi e da 0 a 1 nelle femmine" per la stessa sottospecie. Anche la spinulazione dei femori posteriori risulta assai inferiore nei nostri esemplari rispetto a quella della sottospecie tipica (Tab. 1).

\section{Note Biologiche}

La presenza di una colonia di pipistrelli all'interno della grotta (Rhinolophus ferrunequinum Schreber, 1774) e la conseguente deposizione di guano all'interno della cavità suggerisce che la popolazione di Dolichopoda abbia una dieta saprofaga basata sui resti di artropodi contenuti nel guano. Inoltre l'abbondante materiale vegetale, rami e foglie in decomposizione, che si accumula nei primi metri declivi all'ingresso della Buca Tana non esclude che questi ortotteri si nutrano anche di questi resti visto che entrambi i tipi di alimentazione sono documentati per popolazioni appartenenti a questo genere (De Pasquale et al., 1995) con variazioni legate alla stagionalità e al differente stadio di sviluppo degli esemplari. In almeno un caso ho potuto osservare un episodio di cannibalismo tra individui della stessa specie, comportamento anche questo ben documentato nelle Dolichopoda insieme a quello predatorio.

\section{DisCUSSIONE}

Le specie italiane appartenenti al genere Dolichopoda sono state oggetto, negli ultimi due decenni, di importanti studi nel campo della genetica e della filogenesi molecolare nel tentativo di chiarire i meccanismi di diversificazione indotti dall'evoluzione paleogeografica della penisola e dai relativi mutamenti climatici. Il calcolo dei tempi di divergenza e la messa a punto di orologi molecolari hanno permesso di ricostruire l'albero filogenetico delle varie specie di Dolichopoda e di individuare i loro flussi di migrazione.

Le recenti ricerche di filogeografia (Allegrucci et al., 2011) propongono di collocare la prima colonizzazione della penisola, avvenuta in una sola fase da parte di un antenato ancestrale proveniente da nord-est, tra il medio e il tardo Miocene. Successivamente si sarebbe differenziato il gruppo Dolichopoda laetitiae Dolichopoda geniculata diffondendosi lungo la direttrice appenninica. Da queste specie sarebbero derivate quelle che si trovano oggi nel sud della Penisola (gruppo Dolichopoda capreensis - Dolichopoda palpata) e successivamente, con vari episodi di isolamento e ricolonizzazione, quelle diffuse lungo le coste tirreniche con direttrice da sud verso nord (Dolichopoda schiavazzii - Dolichopoda baccettii - D. aegilion) durante il Pleistocene, in un arco di tempo compreso tra $\mathrm{i}$ 2.4 e 0.6 milioni di anni fa (Allegrucci et al., 2005). Ultimo in ordine di tempo potrebbe essere avvenuto il popolamento della Sardegna (Dolichopoda muceddai) e della Corsica forse in seguito ad un abbassamento del livello marino. All'interno delle varie popolazioni di $D$. schiavazzii è stato osservata una scarsa differenziazione genetica fra quelle che hanno colonizzato ambienti ipogei artificiali (tombe, acquedotti, catacombe, cantine, ecc.) e questo è certamente un risultato prevedibile visto l'adattamento in tempi storici a questi ambienti (Sbordoni, 1985; Minasi et al.,1993).

L'individuazione di una popolazione stabile di Dolichopoda schiavazzii caprai alla Buca Tana di Maggiano, distante almeno un cinquantina di chilo-

Tab. 1. Spinulazione delle zampe: A = Maschi Dolichopoda schiavazzii schiavazzi (miniere abbandonate di Montecastelli, Pomarance, PI); B = femmine D. schiavazzii schiavazzii (miniere di Montecastelli, Pomarance, PI); C = maschi D. schiavazzii caprai (Buca Tana di Maggiano, LU); $\mathrm{D}=$ femmine D. schiavazzii caprai (Buca Tana Maggiano, $\mathrm{LU}$ ); $\mathrm{f}=\mathrm{femore,} \mathrm{t}=\mathrm{tibia}$, ie $=$ margine inferiore esterno, $\mathrm{ii}=$ margine inferiore interno, $\mathrm{se}=$ margine superiore esterno, $\mathrm{si}$ = margine superiore interno.

\begin{tabular}{|c|c|c|c|c|c|c|c|c|c|c|c|c|c|c|c|c|c|c|}
\hline & $\begin{array}{l}\text { 1f } \\
\text { ie }\end{array}$ & $\begin{array}{l}\text { 1f } \\
\text { ii }\end{array}$ & $\begin{array}{l}1 \mathrm{t} \\
\mathrm{se}\end{array}$ & $\begin{array}{l}1 \mathrm{t} \\
\text { si }\end{array}$ & $\begin{array}{l}1 \mathrm{t} \\
\mathrm{ie}\end{array}$ & $\begin{array}{l}\text { 1t } \\
\text { ii }\end{array}$ & $\begin{array}{l}2 \mathrm{f} \\
\mathrm{ie}\end{array}$ & $\begin{array}{l}2 \mathrm{f} \\
\text { ii }\end{array}$ & $\begin{array}{l}2 \mathrm{t} \\
\mathrm{se}\end{array}$ & $\begin{array}{l}2 \mathrm{t} \\
\mathrm{si}\end{array}$ & $\begin{array}{l}2 \mathrm{t} \\
\mathrm{ie}\end{array}$ & $\begin{array}{l}2 \mathrm{t} \\
\text { ii }\end{array}$ & $\begin{array}{l}3 \mathrm{f} \\
\text { ie }\end{array}$ & $\begin{array}{l}3 \mathrm{f} \\
\text { ii }\end{array}$ & $\begin{array}{l}3 \mathrm{t} \\
\mathrm{se}\end{array}$ & $\begin{array}{l}3 \mathrm{t} \\
\mathrm{si}\end{array}$ & $\begin{array}{l}3 \mathrm{t} \\
\mathrm{ie}\end{array}$ & $\begin{array}{l}3 \mathrm{t} \\
\mathrm{ii}\end{array}$ \\
\hline A & 0 & 0 & 2 & 0 & $4-5$ & $4-5$ & 12 & $14-15$ & 9 & 6 & 3 & 6 & 27 & 29 & 23 & 18 & 2 & 1 \\
\hline B & 0 & 0 & 2 & 0 & 4-6 & $5-6$ & $9-12$ & $9-12$ & $6-7$ & 7 & 2 & 2 & $22-31$ & $26-31$ & $21-22$ & $21-22$ & 2 & 2 \\
\hline $\mathrm{C}$ & 0 & 0 & 1 & 0 & $4-5$ & 5 & 0 & 0 & $2-4$ & $3-6$ & $4-5$ & 4 & $11-12$ & $15-20$ & $15-19$ & $14-19$ & $2-3$ & 1 \\
\hline $\mathrm{D}$ & 0 & 0 & 0 & 0 & $4-5$ & $4-5$ & 0 & $0-3$ & 3 & $1-2$ & $3-4$ & $3-4$ & $2-14$ & $4-16$ & $17-20$ & $15-18$ & $2-3$ & 1 \\
\hline
\end{tabular}


metri dall'unica altra località di rinvenimento di questa sottospecie, ovvero la caverna del Fichino di Casciana Terme $(\mathrm{Pi})$, e altrettanto dalla più vicina stazione della specie tipica, cioè gli acquedotti di Livorno (Cisternino, ecc.), pone diversi interrogativi sulle dinamiche di diffusione di D. schiavazzii oltre le due presunte barriere naturali dell'Arno e del Serchio e sull'individuazione della finestra temporale in cui tale migrazione è stata possibile.

É noto che le specie appartenenti al genere $D o-$ lichopoda possono spostarsi in ambiente epigeo, qualora le condizioni favorevoli di umidità e temperatura lo consentano, specialmente nelle ore notturne e in boschi umidi mesofili (Di Russo \& Rampini, 2004). In base a questo presupposto si potrebbe spiegare la colonizzazione di nuove cavità naturali e artificiali, raggiunte attraverso boschi e ambienti epigei favorevoli, da parte di individui adulti in lento e casuale spostamento. Resta comunque da comprendere come sia stato possibile l'attraversamento di possibili barriere naturali come i corsi fluviali dell'Arno e del Serchio rammentando, a questo proposito, che proprio un fiume come il Tevere rappresenta il confine fisico tra due specie, D. laetitiae a nord e D. geniculata geniculata a sud (Di Russo \& Rampini, 2012). É possibile allora, a mio avviso, proporre una ipotesi più suggestiva che renda conto degli spostamenti, avvenuti in tempi più antichi, della specie $D$. schiavazzii a nord dei Monti Livornesi, attraverso i Monti Pisani e quelli di Oltreserchio sino a raggiungere le estreme pendici meridionali del massiccio apuano.

Per quanto riguarda il passaggio di popolazioni di D. schiavazzii da sud (Monti Livornesi) e da sudovest (Monti di Casciana) verso i Monti Pisani si può risalire ad una colonizzazione più antica, risalente almeno al Pleistocene inferiore (tra 1.700 .00 e 700.000 anni fa). In quel periodo il Valdarno inferiore era occupato da una vasta area lacustre posta tra il Monte Albano a nord-ovest e i Monti Pisani ad est e formatasi in seguito ad una serie di sprofondamenti tettonici. Verso sud le colline plioceniche erano maggiormente sollevate e lambivano i Monti Pisani impedendo al mare pleistocenico (sinus pisanus) di invadere le zone lacustri dell'interno (Fig. 4). Questi alti morfologici potevano così costituire un possibile ponte naturale per la penetrazione delle Dolichopoda nell'area dei Monti Pisani. Sembra assodato, in base agli studi geologici e geomorfologici (Federici \& Mazzanti, 1988; Puccinelli, 1991) che l'attuale percorso del fiume Ser- chio costituisse sino a tempi storici solo una diramazione (1'“Auserculus") di un ben più ampio complesso fluviale detto Paleo-Serchio. Nel Pleistocene superiore, durante l'interglaciale Riss-Würm (120.000 80.000 anni fa), questo fiume scorreva in direzione sud-est per poi innestarsi nell'antico alveo del PaleoArno nei pressi dell'attuale abitato di Calcinaia (Pi). Nel I o II stadio della glaciazione Würmiana (tra

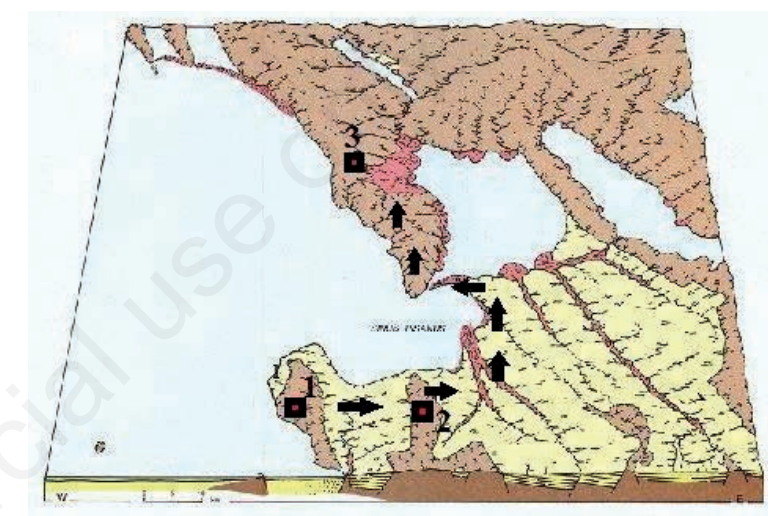

Fig. 4. Schema ricostruttivo della Toscana nord-occidentale nel Pleistocene Inferiore (Federici \& Mazzanti, 1988) e probabile direzione del flusso migratorio di Dolichopoda schiavazzii, 1) Monti Livornesi, 2) Monti di Casciana; 3) Pendici meridionali delle Alpi Apuane.

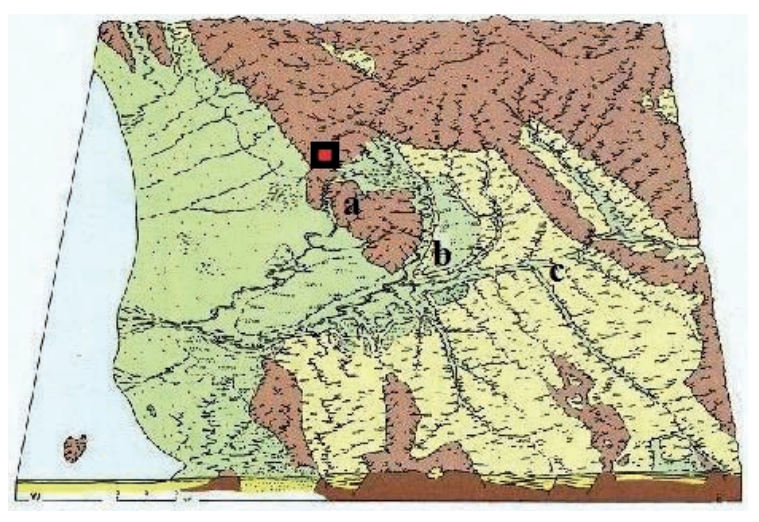

Fig. 5. La Toscana nord-occidentale durante le fasi II e III della glaciazione wurmiana, in a) è indicato il superamento della soglia di Ripafratta da parte di un ramo laterale del Paleo-Serchio, in b) è indicato il corso principale del Paleo-Serchio, in c) il corso del Paleo-Arno (Federici \& Mazzanti, 1988). 
80.000 e 40.000 anni fa), quando i grandi apporti fluviali del Paleo-Serchio colmarono la pianura lucchese, una sua ramificazione iniziò a scorrere verso sudovest, superando la "soglia di Ripafratta", posta tra i Monti Pisani e quelli di Oltreserchio. Nel contempo la pianura pisana e della Versilia si estendeva molto più ad ovest a causa delle trasgressioni marine corrispondenti alle fasi di acme wurmiane che favorirono un abbassamento del livello del mare tra i 60 e i 100 metri rispetto a quello attuale (Fig. 5). Prima della tracimazione alla soglia di Ripafratta, che portò all'istaurarsi dell'attuale corso del Serchio la zona dei monti d'Oltreserchio e la pianura prospiciente, potevano ancora favorire il passaggio di fauna, specialmente nei periodi interglaciali più caldi (Fig. 6) e anche le Dolichopoda avrebbero potuto facilmente ampliare il loro areale, attraverso i Monti Pisani verso nord raggiungendo la zona della Buca Tana di Maggiano. Naturalmente solo uno studio delle affinità genetiche tra la popolazione di $D$. schiavazzii caprai della Buca Tana di Maggiano e quelle geograficamente più vicine potrebbe supportare le mie ipotesi, condotte solo sulle attuali conoscenze paleogeografiche della regione.

\section{CONCLUSIONI}

La nuova popolazione di $D$. schiavazzii caprai individuata alla grotta Buca Tana di Maggiano (Lucca) rappresenta la seconda segnalazione per questa sottospecie dopo quella della Caverna del Fichino di Casciana Terme, distante più di cinquanta chilometri da Lucca e la prima stazione di D. schiavazzii a nord dell'Arno e del Serchio, sulle estreme pendici meridionali del massiccio delle Alpi Apuane. I Monti Pisani e quelli d'Oltreserchio, per la natura dei terreni e il discreto numero di grotte conosciute, se adeguatamente indagati, potrebbero riservare la scoperta di po-

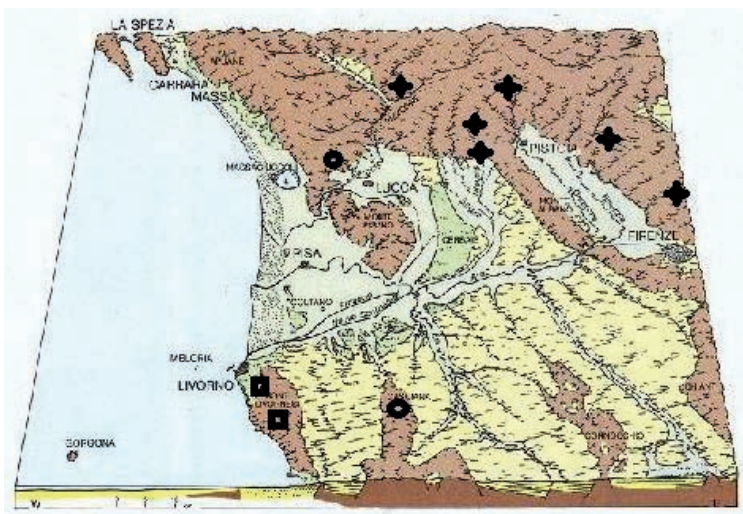

Fig. 6. La Toscana nord-occidentale nell'assetto idrogeologico e morfologico attuale: $n$ stazioni di Dolichopoda schiavazzii schiavazzii, • stazioni di Dolichopoda schiavazzii caprai, $\downarrow$ stazioni di Dolichopoda laetitiae laetitiae.

polazioni intermedie tra quella della Buca Tana e quelle delle vicine colline livornesi. Così non è escluso che D. schiavazzii caprai estenda il suo areale anche verso nord-ovest nelle cavità che si trovano sulle pendici collinari della Versilia. Infine l'esplorazione dei versanti interni delle Alpi Apuane, la Garfagnana e il gruppo montuoso delle Pizzorne a sud-ovest di Lucca servirebbe a meglio definire i confini degli areali tra $D$. schiavazzii caprai e la $D$. laetitiae che pure è segnalata in un'unica stazione in provincia di Lucca (Grotta delle Sane a Bagni di Lucca) e in diverse altre località della limitrofa provincia di Pistoia.

\section{RINGRAZIAMENTI}

Ringrazio Mauro Rampini dell’Università La Sapienza di Roma per i suggerimenti dati nella identificazione della specie.

\section{BIBLIOGRAFIA}

Allegrucci G., Todisco V., Sbordoni V., 2005 - Molecular phylogeography of Dolichopoda cave crickets (Orthoptera, Rhaphidophoridae): a scenario suggested by mitochondrial DNA. Molecular Phylogenetics and Evolution, 37: 153-164.

Allegrucci G., Trucchi E., Sbordoni V., 2011 - Tempo and mode of species diversification in Dolichopoda cave crickets (Orthoptera, Rhaphidophoridae). Molecular Phylogenetics and Evolution, 60: 108-121.

BACCETti B., 1966 - Notulae orthopterologicae, XXI. Le Dolichopoda della Francia e della Spagna. International Journnal of Speleolohy, Lehre, II: 17-28.

BACCETTI B., 1976 - Notulae orthopterologicae. XXXIII. Il popolamento dell'arcipelago toscano. Lavori della Società Italiana di Biogeografia, N.S. 5: 523-540.

Baccetti B., CAPRA F., 1959 - Notulae orthopterologicae. XII. Revisione delle specie italiane del genere Dolichopoda Bol. (Orthopt. Rhaphidophoridae). Redia, XLIV: 165-217.

CAPRA F., 1934 - Una nuova Dolichopoda d'Italia. Bollettino della Società Entomologica Italiana, 46: 40-43. 
De Pasquale L., Cesaroni D., Di Russo C., Sbordoni V., 1995 - Trophic Niche, Age Structure and Seasonaly in Dolichopoda Cave Crickets. Ecography, 18: 217-224

Di Russo C., RAMPINI M., 2004 - Dolichopoda raccolte in ambienti epigei di alcune regioni italiane. Fragmenta Entomologica, Roma, 36: 1-6.

Di Russo C., RAmpini M., 2012 - famiglia Rhaphidophoridae. Fauna d'Italia, Orthoptera. Vol. XLVIII, Calderini, Bologna: 128-129.

Dodero A., 1917 - Materiali per lo studio dei Coleotteri italiani con descrizione di nuove specie. Annali del Museo civico di storia naturale G. Doria, Genova, 47: 377-386.

FEDERICI P.R., MAZZANTI R., 1988 - L'evoluzione della paleogeografia e della rete idrografica del Valdarno inferiore. Bollettino della Società Geografica Italiana, 10-12: 573-615.

LANZA B., 1954 - Speleofauna toscana III. Corologia degli Ortotteri cavernicoli toscani e note sistematiche sul gen. Dolichopoda. Monitore Zoologico italiano, 62: 254-267.

LANZA B., 1957 - Speleofauna toscana IV. Nuove notizie sulla Dolichopoda schiavazzii caprai. Monitore Zoologico italiano, 64: 159.

LazZeroni G., 1970 - Chthonius (s. str.) elongatus, nuova specie cavernicola della Toscana. (Ricerche sugli Pseudoscorpioni, VII). Memorie del Museo Civico di Storia Naturale di Verona, 17: 141-146.

Magrini P. , AbBazzi P., 2011 - Due specie di Raymondionymus della Toscana (Coleoptera, Raymondionymidae) . Fragmenta Entomologica, Roma, 43: 89-106.

Minasi M.G., Allegrucci G., Sbordoni V., 1993 - Population genetic structure in the cave crickets Dolichopoda schiavazzii. Congresso dell'Unione Zoologica Italiana, 55, Riassunti.

PuCCINELli A., 1991 - Nuovi aspetti dell'evoluzione paleogeografica e tettonica al Plio-Quatemario della Piana di Lucca (Toscana). Geografia Fisica e Dinamica Quaternaria, 171-177.

Sbordoni V., Allegrucci G., Cesaroni D., Cobolli Sbordoni M., De Mattheis E., 1985 - Genetic structure of populations and species of Dolichopoda cave crickets: evidence of peripatric divergence. In: SBordoni, V. (Ed.), Genetics and ecology incontact zones of populations. Bollettino di Zoologia, 52: 95-114.

VANNI S., 1987 - Catalogo corologico dei Duvalius della Toscana (Coleoptera, Carabidae, Trechinae). Atti della Società Toscana di Scienze Naturali, Memorie, serie B, 94: 271-291. 